\title{
A Survey of Young Stars in Taurus for Multiplicity
}

\author{
CH. LEINERT, N. WEITZEL, M. HAAS, R. LENZEN \\ Max-Planck-Institut für Astronomie, Königstuhl, \\ D-6900 Heidelberg 1, Germany \\ H. ZINNECKER
}

Institut für Astronomie und Astrophysik der Universität Würzburg, Am Hubland, D-8700, Würzburg, Germany

\section{J. CHRISTOU}

Steward Observatory, University of Arizona, Tucson AZ, 85721, USA

\section{S. T. RIDGWAY}

National Optical Astronomy Observatories, P.O. Box 26732, Tucson AZ, 85726, USA

\section{R. JAMESON}

Astronomy Group, University of Leicester, University Road, Leicester LE1 7RH, England

\section{A. RICHICHI \\ Osservatorio Astrofisico di Arcetri, Largo Enrico Fermi 5, I-50125 Firenze, Italy}

\begin{abstract}
We surveyed all stars in Taurus $\left(3^{h} 45^{m}<\alpha<4^{h} 15^{m}, 15^{\circ}<\delta<\right.$ $35^{\circ}$ ) for multiplicity which are contained in the Herbig-Bell catalogue of young stars and have a 2 micron brightness of $K \leq 9.5$ mag. This sample consists of 106 stellar systems (single or multiple), of which $4 \overline{3}$ are double or multiple according to the criteria of our survey, i.e. with separations of $\approx 0^{\prime \prime} .2 \leq \mathrm{d} \leq 10^{\prime \prime}$. Of these, 23 binaries are new detections found in this survey. The resulting degree of multiplicity, $43 / 106=41 \pm 6 \%$, is higher than found for main-sequence stars. Provided that the period distribution is the same for young stars as on the main sequence, our result implies that the vast majority of stars are born in binary or multiple systems.
\end{abstract}

\section{GOALS OF THE SURVEY}

Although the majority of stars are members of binary or multiple systems, the formation of such systems is still not well understood. Indeed this question represents one of the major unsolved problems in stellar astronomy. A clear review of the current status of theoretical work has recently been given by Boss (1992). At present, observations do not allow us to discriminate between the different proposed mechanisms of formation (e.g., capture of a companion, fragmentation during the collapse phase or instabilities in a massive circumstellar disk). Guided by the assumption that differences between competing formation 
mechanisms may show most clearly in the youngest binaries, we decided in this situation to survey the young stars in the nearby Taurus-Auriga star forming region (distance $=140 \mathrm{pc}$, Elias 1978) for multiplicity.

The first goal of this survey is to simply find the percentage of binary or multiple systems among the young stars in this region, including a minimum of physical (e.g., brightness ratio) and orbital information (separation, position angles). This limited data base already will serve as a check on the predictions of the competing theories of binary star formation. In addition, the comparison of our data with results obtained for main-sequence stars (Abt 1983, 1987; Duquennoy \& Mayor 1991) may show differences which — if not due to incompleteness in one or both of the data sets - should be due to temporal evolution.

A second goal, although not addressed here, is to determine broadband spectra for the individual components of the detected binaries, yielding estimates of luminosities and relative masses, and possibly showing luminous infrared companions in addition to those found earlier, as T Tau (Dyck et al. 1982), Glass I (Chelli et al. 1988), Haro 6-10 (Leinert \& Haas 1989a) or XZ Tau (Haas et al. 1990). Finally, following the orbits of the closer binaries will in the long run allow us to obtain direct measurements of the masses of young stars, a crucial test for the calculations of early stellar evolution.

\section{OBSERVATIONS}

For our observations we chose the technique of near-infrared speckle interferometry, both with a $2 \mathrm{D}$ camera and with $1 \mathrm{D}$ slit scanning systems, supplemented by near-infrared lunar occultation observations as far as occultation events happened to occur. The sensitivity is similar for the three methods with a limiting magnitude for observations at a 3-4 m telescope of $K=9-9.5 \mathrm{mag}$; the spatial resolution, of course, is highest for the occultation observations.

The near-infrared is a natural choice of the wavelength band, since most of the young stars are of spectral type $M$ and late $K$, several are substantially reddened by extinction, and many show additional infrared emission due to circumstellar disks. The speckle technique is well adapted to the problem, since the most probable binary separations, $10-100 \mathrm{AU}$ for main sequence stars, or $0 " .07-0 \prime .7$ at the distance of the Taurus clouds, mostly are within the reach of this method, the diffraction limit being $0 " 13$ for observations in $K$ at a $3.5 \mathrm{~m}$ telescope.

Most of the observations were obtained on the $3.5 \mathrm{~m}$ telescope on Calar Alto, with the 1D slit scanning system (Leinert \& Haas 1989b) mounted in September 1991 and February 1992 and with the 2D camera (Lenzen et al. 1990) in October 1991 and March 1992. A large part of the data also was obtained with the NOAO speckle camera (Beckers et al. 1988) on the Kitt Peak $4 \mathrm{~m}$ telescope in December 1990 and December 1991. In addition lunar occultations of seven young stars (HK Tau A, FY Tau, AA Tau, LkHa 331, HK Tau/G2, IW Tau, and Haro 6-10) were observed with a fast integrating photometer (Adams et al. 1988) at the 4.2 m William Herschel Telescope on La Palma on September 23, 1991. As a rule, objects with complete published results of near-infrared speckle interferometry or lunar occultations were not observed again.

In summary, our set of measurements is inhomogeneous in telescopes and 
instruments used and in the sources from which the results are drawn. On the other hand, we do not consider this to present a problem, since speckle observations always tend to form inhomogeneous data sets because of their strong dependence on the degree of atmospheric disturbance. While the speckle observations cover an area of only $5^{\prime \prime} \times 5^{\prime \prime}$ to $8^{\prime \prime} \times 10^{\prime \prime}$ around the individual objects, imaging of a wider surrounding $\left(\approx 1^{\prime} \times 1^{\prime}\right)$ was available for 52 of them, which is about half of the surveyed stars (Moneti \& Zinnecker 1991, Weintraub 1989, Simon et al. 1992 and private communication).

\section{DEFINITION OF THE SURVEY}

The defining criteria for the survey are the following:

Sky region

Object list

Wavelength

Brightness limit

Separations
Taurus-Auriga $\left(3^{h} 45^{m}<\alpha<5^{h} 15^{m}, 15^{\circ}<\delta<35^{\circ}\right)$

Young stars with optical slit spectra as compiled

in the Herbig-Bell catalogue (1988)

$$
2.2 \text { micron }
$$

$\mathrm{K} \leq 9.5 \mathrm{mag}$

$0^{\prime \prime} .13$ (diffraction limit) $-10^{\prime \prime}$

Excluding the two Herbig Ae/Be stars Elias 1 and $A B$ Aur, there remain 106 systems of young low-mass stars in the survey, of which about two thirds have been classified as classical $\mathrm{T}$ Tauri stars, about one third as weak line $\mathrm{T}$ Tauri stars, and of which $10 \%$ lack classification. Our task was to observe those 93 targets not sufficiently covered by published results.

This survey is not complete in the strict sense of the word, since the target list certainly does not contain all young stars of the Taurus-Auriga cloud complex, but only those known at the time of catalogue compilation with sufficient spectral information. Also, since most of the stars in the survey are variable this is one well-known characteristic feature of stellar youth - setting a brightness limit based on published observations, which often include only one epoch, is somewhat arbitrary. In addition we may miss companions both at small and large separations (because we do not reach the diffraction limit for weaker objects, or because imaging of the surroundings is missing in many cases). However, to express our expectation that the survey nevertheless represents a statistically meaningful sample, we call it quasi-complete.

\section{RESULTS}

The results presented here represent the status of the survey as of March 1992. They are provisional in the sense that small corrections may still be applied to the data. A full presentation of the final results is being prepared for publication in Astronomy and Astrophysics.

In our survey we found 23 new binary systems. These are presented in Table 1. In addition, results on 20 binaries meeting our criteria had already been published. These are summarised in Table 2. IS Tau, IQ Tau and DH Tau were considered as single stars because they were unresolved in more than one lunar occultation observation (Simon et al. 1992, Chen et al. 1990), HL Tau, 
DG Tau and L1551 IRS5 because they showed a halo only in previous speckle observations (Beckwith et al. 1984, Leinert and Haas 1988, Leinert et al. 1991), while V826 Tau (Mundt et al. 1983) and HBC 427 (Mathieu et al. 1989) qualify as unresolved for the purposes of our survey because their separation falls below our lower limit. In total then, out of 106 young stellar systems we find 43 to be multiple, among which there are 39 binaries, three triples (UX Tau, UZ Tau and HV Tau, if we count here the close companion), and one quadruple system, GG Tau. Taken at face value these data imply a rather high degree of multiplicity of $43 / 106=41 \pm 6 \%$ for young low-mass stars in Taurus, although we only count systems with projected component separations in the range $0^{\prime \prime} .13-10^{\prime \prime}$.

TABLE 1. New pre-main sequence binaries in Taurus

\begin{tabular}{|c|c|c|c|c|c|c|}
\hline $\begin{array}{c}\text { HBC } \\
\text { No. }\end{array}$ & Name & $\underset{(\mathrm{mag})}{\mathbf{K}}$ & $d\left(^{\prime \prime}\right)$ & $\begin{array}{l}\text { Position } \\
\text { angle }\left({ }^{\circ}\right)\end{array}$ & $\begin{array}{l}\text { Brightness } \\
\text { ratio }\end{array}$ & Rem \\
\hline 351 & $034903+2431$ & 9.15 & $0.61 \pm 0.03$ & $317 \pm 3$ & $0.22 \pm 0.02$ & \\
\hline 358 & $040047+2603 W$ & 9.40 & $1.58 \pm 0.03$ & $226 \pm 1$ & $0.60 \pm 0.05$ & \\
\hline 367 & V773 Tau & 6.41 & $0.17 \pm 0.01$ & $295 \pm 3$ & $0.13 \pm 0.04$ & \\
\hline 368 & $\mathrm{LkCa} 3$ & 7.53 & $0.47 \pm 0.04$ & $78 \pm 1$ & $0.68 \pm 0.05$ & \\
\hline 369 & FO Tau & 8.13 & $0.165 \pm 0.005$ & $180 \pm 4$ & $0.92 \pm 0.04$ & 1 \\
\hline 30 & DD Tau & 7.93 & $0.57 \pm 0.03$ & $188 \pm 2$ & $0.64 \pm 0.01$ & \\
\hline 31 & CZ Tau & 9.30 & $0.33 \pm 0.01$ & $84 \pm 3$ & $0.46 \pm 0.03$ & \\
\hline 377 & FQ Tau & 9.28 & $0.79 \pm 0.01$ & $69 \pm 1$ & $0.90 \pm 0.01$ & \\
\hline 379 & $\mathrm{LkCa} 7$ & 8.33 & $1.05 \pm 0.01$ & $25 \pm 2$ & $0.56 \pm 0.02$ & \\
\hline 44 & FX Tau & 8.09 & $0.91 \pm 0.01$ & $292 \pm 3$ & $0.55 \pm 0.01$ & \\
\hline 47 & LkHa 331 & 8.66 & $0.30 \pm 0.01$ & $290 \pm 4$ & $0.73 \pm 0.04$ & \\
\hline 398 & HK Tau/G2 & 8.05 & $0.18 \pm 0.01$ & $300 \pm 4$ & $0.88 \pm 0.03$ & 2 \\
\hline 55 & GH Tau & 7.61 & $0.35 \pm 0.01$ & $120 \pm 1$ & $0.91 \pm 0.05$ & \\
\hline 404 & Elias 12 & 6.94 & $0.41 \pm 0.01$ & $332 \pm 1$ & $0.46 \pm 0.02$ & \\
\hline 411 & CoKu Tau/3 & 8.25 & $2.04 \pm 0.07$ & $177 \pm 3$ & $0.29 \pm 0.02$ & \\
\hline 412 & $043230+174$ & 9.10 & $0.70 \pm 0.01$ & $68 \pm 1$ & $1.00 \pm 0.02$ & \\
\hline 416 & Haro 6-28 & 9.26 & $0.66 \pm 0.02$ & $246 \pm 1$ & $0.63 \pm 0.03$ & \\
\hline 68 & VY Tau & 8.98 & $0.66 \pm 0.02$ & $317 \pm 2$ & $0.26 \pm 0.02$ & \\
\hline 422 & LkHa 332/G2 & 8.13 & $0.30 \pm 0.01$ & $243 \pm 2$ & $0.60 \pm 0.05$ & \\
\hline 423 & LkHa 332/G1 & 7.31 & $0.23 \pm 0.02$ & $85 \pm 2$ & $0.58 \pm 0.03$ & \\
\hline 69 & LkHa 332 & 7.73 & $0.33 \pm 0.03$ & $204 \pm 2$ & $0.22 \pm 0.03$ & \\
\hline 420 & IW Tau & 8.33 & $0.27 \pm 0.02$ & $177 \pm 2$ & $0.91 \pm 0.04$ & 1 \\
\hline 76 & UY Aur & 6.93 & $0.89 \pm 0.01$ & $225 \pm 1$ & $0.28 \pm 0.02$ & 3 \\
\hline
\end{tabular}

Remarks: (1) Position angle $0^{\circ}$ possible as well;

(2) First found by lunar occultation observations;

(3) See also Weintraub (1989) who observed a brightness ratio of 0.45 and a north-south separation of $0^{\prime \prime} .70$.

Among possible biases increasing the observed degree of duplicity are false detections due to misinterpretation of measured data or due to chance projections and the fact that binaries, because of the light added by the companion, always are overrepresented in a magnitude-limited sample. On the other hand, our ability to detect companions may suffer in the case of faint objects, for projected separations not considerably larger than the diffraction limit, and for 
small brightness ratios (secondary/primary) of the components. None of these effects is expected to be large, and they tend to cancel each other. Therefore we do not apply any correction factors to our data.

TABLE 2. Known pre-main sequence binaries in Taurus

\begin{tabular}{|c|c|c|c|c|c|c|c|}
\hline $\begin{array}{l}\text { HBC } \\
\text { No. }\end{array}$ & Name & $\underset{\text { (mag) }}{\mathrm{K}}$ & & $d(")$ & $\begin{array}{l}\text { Position } \\
\text { angle }\left({ }^{\circ}\right)\end{array}$ & $\begin{array}{l}\text { Brightness } \\
\text { ratio }\end{array}$ & Ref \\
\hline $\begin{array}{l}352 / 353 \\
360 / 361\end{array}$ & $\begin{array}{l}035120+3154 \\
040142+2150\end{array}$ & $\begin{array}{l}9.03 \\
9.36\end{array}$ & $\begin{array}{l}8.6 \\
7.2\end{array}$ & $\begin{array}{l} \pm 0.8 \\
\pm 0.8\end{array}$ & $\begin{array}{l}62 \pm 5 \\
74 \pm 5\end{array}$ & $\begin{array}{l}0.77 \pm 0.05 \\
0.91 \pm 0.05\end{array}$ & $\begin{array}{l}1 \\
1\end{array}$ \\
\hline 383 & FS Tau & 8.03 & 0.265 & $5 \pm 0.005$ & $60 \pm 5$ & $0.12 \pm 0.01$ & 2 \\
\hline 35 & T Tau & 5.6 & 0.73 & \pm 0.03 & $176 \pm 1$ & $0.16 \pm 0.005$ & 3 \\
\hline 386 & FV Tau & 7.48 & 0.72 & \pm 0.10 & $270 \pm 5$ & $0.83 \pm 0.05$ & 2 \\
\hline 387 & FV Tau/c & 8.88 & 0.74 & \pm 0.14 & $293 \pm 3$ & $0.17 \pm 0.02$ & 2 \\
\hline 389 & Haro 6-10 & 6.98 & 1.21 & \pm 0.04 & $355 \pm 1$ & $0.13 \pm 0.01$ & 4 \\
\hline $42 / 43$ & $\begin{array}{r}\text { UX Tau AC } \\
A B\end{array}$ & 7.29 & $\begin{array}{l}2.7 \\
5.9\end{array}$ & $\begin{array}{l} \pm 0.1 \\
\pm 0.1\end{array}$ & $\begin{array}{l}181 \pm 2 \\
269 \pm 2\end{array}$ & $\begin{array}{l}0.069 \pm 0.01 \\
0.27 \pm 0.03\end{array}$ & $\begin{array}{l}6 \\
6\end{array}$ \\
\hline 45 & DK Tau & 7.00 & 2.8 & \pm 0.3 & $115 \pm 7$ & $0.25 \pm 0.10$ & 5 \\
\hline 48 & HK Tau & 8.38 & 2.4 & \pm 0.1 & $175 \pm 2$ & $0.059 \pm 0.01$ & 6 \\
\hline 50 & XZ Tau & 8.17 & 0.30 & \pm 0.02 & $154 \pm 3$ & $0.35 \pm 0.02$ & 7 \\
\hline $51 / 395$ & V710 Tau & 8.14 & 3.24 & \pm 0.10 & $357 \pm 1$ & $0.83 \pm 0.01$ & 8 \\
\hline $52 / 53$ & $\underset{\text { ew }}{\text { UZ Tau w }}$ & $\begin{array}{l}8.06 \\
6.86\end{array}$ & $\begin{array}{l}0.34 \\
3.78\end{array}$ & $\begin{array}{l} \pm 0.06 \\
\pm 0.07\end{array}$ & $\begin{array}{r}0 \pm 8 \\
273 \pm 1\end{array}$ & $\begin{array}{l}0.36 \pm 0.05 \\
0.50 \pm 0.05\end{array}$ & $\begin{array}{l}2 \\
2\end{array}$ \\
\hline 54 & $\begin{array}{r}\text { GG Tau Aa } \\
A B\end{array}$ & 7.19 & $\begin{array}{l}0.26 \\
10.3\end{array}$ & $\begin{array}{l} \pm 0.01 \\
\pm 0.2\end{array}$ & $\begin{array}{r}9 \pm 2 \\
184 \pm 2\end{array}$ & $\begin{array}{l}0.64 \pm 0.01 \\
0.10 \pm 0.02\end{array}$ & $\begin{array}{l}9 \\
6\end{array}$ \\
\hline & $\mathbf{B b}$ & & 1.4 & \pm 0.2 & $135 \pm 5$ & $0.19 \pm 0.02$ & 9 \\
\hline $60 / 406$ & HN Tau & 8.14 & 3.1 & \pm 0.1 & $215 \pm 2$ & $0.042 \pm 0.01$ & 6 \\
\hline $414 / 415$ & HP Tau/G2 & 6.74 & 9.9 & \pm 0.1 & $245 \pm 1$ & $0.24 \pm 0.03$ & 6 \\
\hline 418 & HV Tau Aa & 7.75 & $\begin{array}{l}0.035 \\
4.00\end{array}$ & $\begin{array}{l}5 \pm 0.002 \\
\pm 0.40\end{array}$ & $\begin{array}{c}273 \pm 13 \\
45 \pm 5\end{array}$ & $\begin{array}{l}0.58 \pm 0.05 \\
0.029 \pm 0.002\end{array}$ & $\begin{array}{l}2 \\
2\end{array}$ \\
\hline $73 / 424$ & Haro 6-37 & 7.31 & 2.7 & \pm 0.1 & $37 \pm 2$ & $0.45 \pm 0.03$ & 6 \\
\hline 75 & DS Tau & 8.26 & 7.1 & \pm 0.1 & $294 \pm 2$ & $0.045 \pm 0.01$ & 6 \\
\hline $80 / 81$ & RW Aur & 6.87 & 1.50 & \pm 0.01 & $258 \pm 1$ & $0.23 \pm 0.01$ & 8 \\
\hline
\end{tabular}

References: (1) Herbig-Bell catalogue (1988)

(2) Simon et al. (1992)

(3) Ghez et al. (1991)

(4) Leinert \& Haas (1989a)

(5) Weintraub (1989)
(6) Moneti \& Zinnecker (1991)

(7) Haas et al. (1990)

(8) This work

(9) Leinert et al. (1991

Concerning the reduced activity of weak line $\mathrm{T}$ Tauri stars, the suspicion has repeatedly been meantioned that it might be due to the presence of a companion which disturbs or disrupts the circumstellar disk of the young star. Though this statement still may be true, we find no general support for it within our survey. The degree of mulitiplicity is not significantly different between the classical $\mathrm{T}$

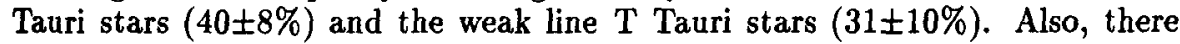
appear to be no significant differences in the degree of multiplicity of young stars between the different substructures of the Taurus-Auriga cloud complex. 


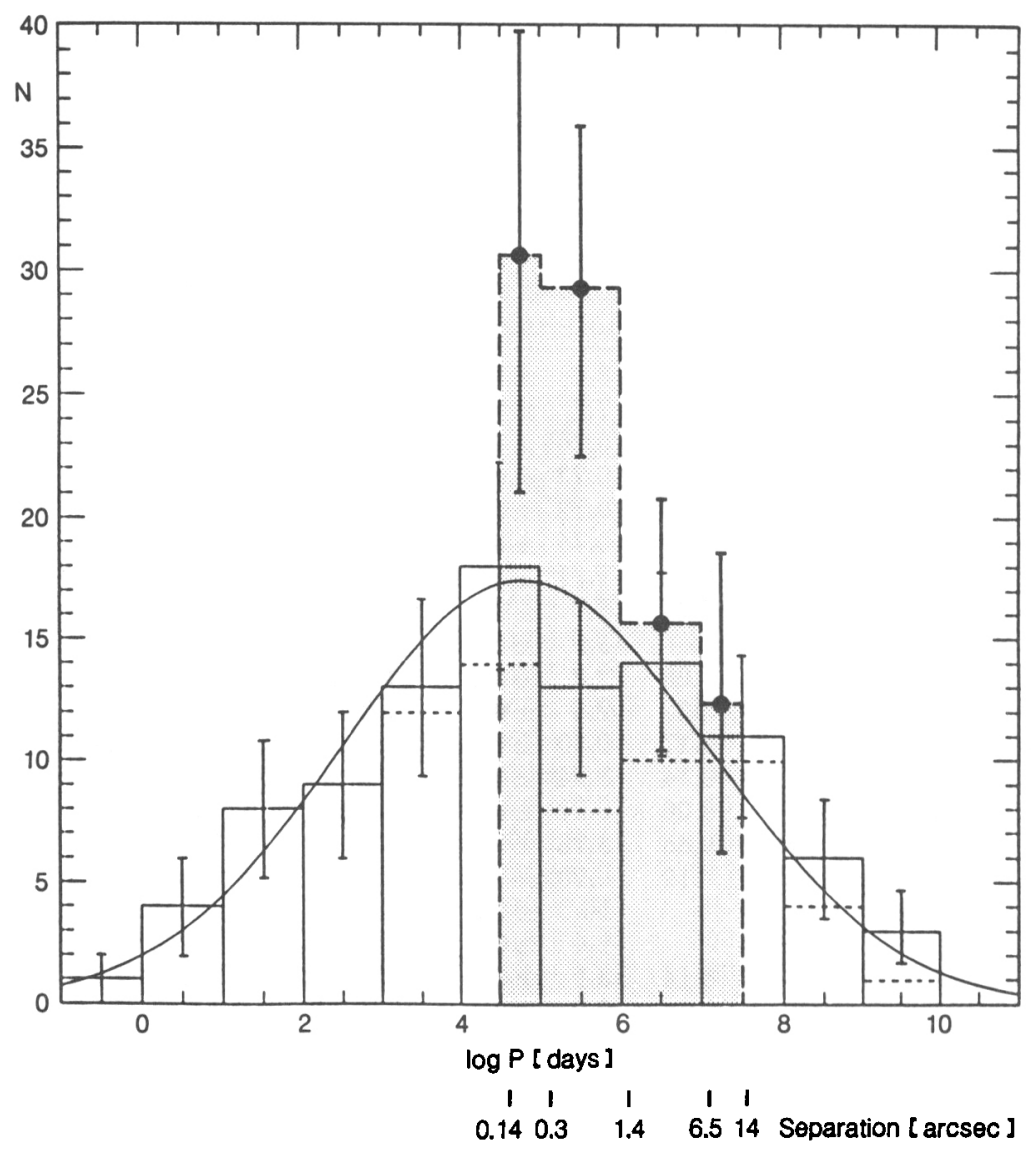

FIGURE 1. Comparison of our data on duplicity of young stars (broken line, shaded area) with the observations of nearby solar type stars by Duquennoy \& Mayor (1991) as reproduced from their paper. For this comparison we have converted measured angular separations into orbital periods $P$ as indicated in the bottom line of the Figure. $N$ is the number of binaries the periods of which fall into a given interval.

\section{COMPARISON WITH THE MULTIPLICITY OF MAIN-SEQUENCE STARS}

The multiplicity of nearby solar type stars (Duquennoy \& Mayor 1991) as well as that of $F$ and $G$ type stars (Abt 1983, 1987) has extensively been studied, with essentially the same result: the degree of multiplicity is close to $60 \%$. Here we compare our data to the more recent work of Duquennoy and Mayor. Since these authors present their results as a period distribution, we also convert our measured separations to periods, using a system mass of $1 \mathcal{M}_{\odot}$, a distance to the Taurus-Auriga clouds of $140 \mathrm{pc}$ and the simplifying assumption that the ob- 
served projected separation equals the semimajor axis of the system. We neglect the - in the average - moderate underestimate of semima jor axis introduced by the last assumption. Our resulting period distribution is overplotted over the results of Duquennoy and Mayor in Figure 1. In the range of periods common to both studies, corresponding to observed separations of $0^{\prime \prime} .14-14^{\prime \prime}$, the degree of duplicity we found is higher by a factor of $1.6 \pm 0.3$ than the result for solar type main-sequence stars. This $2 \sigma$-effect, if confirmed by future work, has important consequences for the multiplicity of young stars: with the assumption that young stars do have the same overall period distribution as their solar type main-sequence counterparts the extrapolation from our measured to the total degree of duplicity for young stars, including all possible periods from days to millions of years, then sums up to $98 \pm 23 \%$. This high value suggests again that we cannot have missed many young binaries in our survey.

\section{CONCLUSION}

The results of our survey are consistent with the concept and support the idea that the large majority if not almost all stars are born in binary or multiple systems. We interpret - as others do - this preference for multiplicity as a consequence of conservation of angular momentum during the early phases of stellar formation. In any case, a high degree of multiplicity removes at least part of the anglar momentum problem in star formation theories. Single star formation may be a process even more uncommon than generally thought.

\section{REFERENCES}

Abt, H.A. 1983, ARA\&A, 21, 243

Abt, H.A. 1987, $A p J, 317,353$

Adams, D.J., Becklin, E.E., Jameson, R.F., Longmore, R.F, Sandquist, A. A., \& Valentijn, E. $1988, A p J, 327$, L65

Beckers, J.M., Christou, J.C., Probst, R.G., Ridgway, St., \& von der Lühe, O. 1988, in High-Resolution Imaging by Interferometry, ESO Conf. Proc. No. 29, ed. F. Merkle, Garching, p. 393

Beckwith, S., Zuckerman, B., Skrutskie, M.F., \& Dyck, H.M. 1984, ApJ, 287, 793

Boss, A.P. 1992, in Close Binary Stars, J. Sahade, G. McClusky, and Y. Kondo, eds., Kluwer, in press

Chelli, A., Zinnecker, H., Carrasco, L., Cruz-Gonzales, I., \& Perrier, Ch. 1988, $A \& A$, 207, 46

Chen, W., Simon, M., Longmore, A., Howell, R., \& Benson, J. 1990, ApJ, 357, 224

Duquennoy, A. \& Mayor, M. $1991 A \& A, 248,485$

Dyck, H.M., Simon, T., \& Zuckerman, B. 1982, $A p J, 255, \mathrm{~L} 103$

Elias, J.H. 1978, $A p J, 224,857$

Ghes, A.M., Neugebauer, G., Gorham, P.W., Haniff, C.A., Kulkarni, S.R., Mattheuws, K., Koresko, C.D., \& Beckwith, S.V.W. 1991, AJ, 102, 2066

Haas, M., Leinert, Ch., \& Zinnecker, H. 1990, $A \& A, 230$, L1

Herbig, G.H. \& Bell, K.R. 1988, Lick Obs. Bull., No. 1111

Leinert, Ch. \& Haas, M. 1988, in High-Resolution Imaging by Interferometry, ESO Conf. Proc. No. 29, ed. F. Merkle, Garching, p.533

Leinert, Ch. \& Haas, M. 1989a, ApJ, 342, L39

Leinert, Ch. \& Haas, M. 1989b, $A \& A, 221,110$

Leinert, Ch., Haas, M., Richichi, A., Zinnecker, H., \& Mundt, R. 1991, $A \mathcal{E} A, 250,407$ 
Lenzen, R., Hippler, St., \& Vogel, A. 1990, Astron. Ges. Abstr. Ser., 5, 99

Mathieu, R.D., Walter, F.M., \& Myers, P.C. 1989, $A J, 98,987$

Moneti, A. \& Zinnecker, H. 1991, $A \& A, 242,428$

Mundt, R., Walter, F.M., Feigelson, E.D., Finkenzeller, U., Herbig, G.H., \& Odell, A.P. 1983, ApJ, 269, 229

Simon, M., Chen, W.P., Howell, R.R., Benson, J.A., Slowik, D. 1992 ApJ, 384, 212

Weintraub, D.A. 1989, thesis, Univ. of California Los Angeles

\section{DISCUSSION}

MAZEH: How did you transform a separation to a period? What eccentricity distribution did you use?

LEINERT: We assumed that the observed separation is equal to the semimajor axis of the orbit, and that the combined system mass is equal 1 solar mass. For an eccentricity distribution of $f(e)=2 e$, as appropriate for wide mainsequence systems, the first assumption is off by only $10 \%$ since the shortening effect by projection and the increase by eccentricity partly cancel.

LATHAM: Is it possible that some of your companions might be very young and therefore luminous brown dwarfs? They would fade away and become too faint to detect as companions, and would have too low a mass to be discovered spectroscopically, to be discovered around main sequence stars.

LEINERT: The problem is in the comparatively large distance to Taurus of 140 pc. According to Burrows, Hubbard, \& Lunine (ApJ, 345, 939, 1989) a brown dwarf of $0.06 \mathcal{M}_{\odot}$ at the age of $10^{8}$ years has a luminosity of $10^{-3} \mathrm{~L}_{\odot}$. For an $M$ dwarf near the end of the main sequence, this luminosity would correspond to a $K$ brightness of $+14.6,4$ magnitudes below our detection limit. However, the evolutionary calculations of d'Antona \& Mazzibelli $(A p J, 296,502,1985)$ show very young brown dwarfs of this mass to have 100 times higher luminosity from $10^{4}$ to $10^{5}$ years with a decline by only a factor of three to the end of the deuterium burning at $\sim 2 \times 10^{6}$ years. Yes, marginally we might be able to see very young brown dwarfs in our survey.

MAYOR: What is the typical mass of your objects? The reason for this question is: when comparing the rate of binaries from $M$ dwarfs to $G$ dwarfs in the solar vicinity and the red giant stars in open clusters (for $M_{1} \sim 2-3 \mathcal{M}_{\odot}$ ) we observe an increase of this rate. I believe it is important to compare the binary rate to a sample of comparable primary mass.

LEINERT: The typical mass range of our ob jects may be 0.4 to slightly over $1.0 \mathcal{M}_{\odot}$. For a meaningful comparison of duplicities between pre-main sequence stars and main sequence stars, a sample of main sequence stars matching the mass distribution of the young stars as closely as possible should be put together.

CLARKE: What fraction of your stars were outside subgroups and what was the binary frequency for these 'isolated' stars?

LEINERT: We tend to be generous when assigning a star as a member of a subgroup, making it a somewhat arbitrary process so far. This leaves only 8 stars 'isolated', of which 5 happen to be double systems. But, we caution that these are small numbers on a somewhat arbitrary sample. 
BOSS: You estimated that no more than about $5 \%$ of your companions could be physically unassociated with the primaries. Did this estimate include both stars within the Taurus subgroup, as well as background $\mathrm{M}$ and $\mathrm{K}$ dwarfs?

LEINERT: Yes, it is based on M. Simon's imaging of the surroundings of observed young stars. The derived areal density of stars to a given brightness limit includes all types of stars.

MATHIEU: (Question directed to Helmut Abt:) Several talks this morning have suggested that all $\mathrm{T}$ Tauri stars are multiple. When incompleteness corrections are applied to the main-sequence binary observations, what is the inferred total main-sequence binary frequency?

ABT: It is easy to show that at least $60-70 \%$ of the primaries have secondaries. Beyond that it depends on one's courage in extrapolating from secondaries that we can detect spectroscopically to ones that we cannot detect.

EVANS: I just finished a survey of Cepheids with IUE. It appears from this that there are also more companions than predicted from B star results for long binary periods. 